\title{
Post-Newtonian Kinetic Theory
}

\author{
Gilberto M. Kremer, ${ }^{1, *}$ \\ ${ }^{1}$ Departamento de Física, Universidade Federal do Paraná, Curitiba 81531-980, Brazil
}

\begin{abstract}
A kinetic theory for relativistic gases in the presence of gravitational fields is developed in the second post-Newtonian approximation. The corresponding Boltzmann equation is determined from the evolution of the one-particle distribution function with respect to the proper time along the world line of the particle. From the knowledge of the equilibrium Maxwell-Jüttner distribution function in the second post-Newtonian approximation the components of the particle four-flow and energy-momentum tensor are obtained. The Eulerian hydrodynamic equations for the mass density, mass-energy density and momentum density in the second post-Newtonian approximation are determined from the Boltzmann equation. It is shown that the combination of the hydrodynamic equations of mass and mass-energy densities leads to the hydrodynamic equation for the internal energy density in the first post-Newtonian approximation.
\end{abstract}

\section{INTRODUCTION}

Post-Newtonian theory is a method of successive approximations in powers of $1 / c^{2}$ for the solution of Einstein's field equations which was proposed by Einstein, Infeld and Hoffmann [1] in 1938. In this method the components of the metric tensor in the order $\mathcal{O}\left(c^{n}\right)$ which appear in the Ricci tensor of Einstein's field equations are determined from the knowledge of the energy-momentum tensor in the order $\mathcal{O}\left(c^{n-2}\right)$. The first post-Newtonian hydrodynamic equations were determined by Chandrasekhar [2] and Weinberg [3] and the second post-Newtonian hydrodynamic equations by Chandrasekhar and Nutku [4].

The derivation of hydrodynamic equations from a transfer equation for arbitrary macroscopic quantities which are associated with mean values of microscopic quantities is an old subject in the literature of kinetic theory of gases which goes back to the work of Maxwell [5] in 1867. In 1911 Enskog [6] determined from the Boltzmann equation a general transfer equation for an arbitrary function of the space-time and particle velocity where the hydrodynamic equations can be obtained.

The main objective of this work is to develop a kinetic theory of gases within the framework of the second postNewtonian approximation. The first post-Newtonian version of Boltzmann equation was proposed in the works [7, 8]. Here the second post-Newtonian approximation for the Boltzmann equation is determined from the evolution of the one-particle distribution function with respect to the proper time along the world line of the particle.

In equilibrium the relativistic gas is characterized by the Maxwell-Jüttner distribution function (see e.g. 9]) here its expression in the second post-Newtonian approximation is obtained from the components of the metric tensor and of the hydrodynamic and particle four-velocities in the same order. From the knowledge of the Maxwell-Jüttner distribution function it is possible to determine the second post-Newtonian approximation of the particle four-flow and energy-momentum tensor components through integration of their expressions which are defined in terms of the one-particle distribution function. The resulting expressions correspond to those obtained from a phenomenological theory based on their decomposition in terms of the hydrodynamic four-velocity. The components of the particle fourflow and energy-momentum tensor in the first post-Newtonian approximation from the Maxwell-Jüttner distribution function were determined in [10].

From the usual method in the kinetic theory of gases the hydrodynamic equations for the mass density, mass-energy density and momentum density are obtained from the integration of the Boltzmann equation when it is multiplied with the rest mass and the components of the particle momentum four-vector - which corresponds in the non-relativistic case to the energy and momentum of a particle - respectively. Due to the conservation laws of the particle rest mass and momentum four-vector these quantities are identified as summational invariants which imply vanishing production terms related to the collision operator of the Boltzmann equation. The Eulerian hydrodynamic equations derived from the post-Newtonian Boltzmann equation correspond to those obtained from the phenomenological theory which follow from the conservation equations of the particle four-flow and energy-momentum tensor [4]. By neglecting the relativistic corrections the hydrodynamic equations for the mass and mass-energy densities coincide and correspond to the Newtonian continuity equation. However, their difference leads to the hydrodynamic equation for the internal energy density, which is an expression in the first post-Newtonian approximation. This result is compatible with

\footnotetext{
*kremer@fisica.ufpr.br
} 
energy conservation law in the post-Newtonian theory [2, 4, 11], since the first post-Newtonian expression for the energy conservation law follows only from the knowledge of the second post-Newtonian approximation.

The paper is outlined as follows: in Section II we introduce the main results from the second post-Newtonian approximation theory that will be used in the paper. The determination of the second post-Newtonian Boltzmann equation, Maxwell-Jüttner distribution function and Eulerian hydrodynamic equations are the subject of the Sections [II] [IV] and [V] respectively. The conclusions of the work are stated in the last section. The notations used here are: Greek indices take the values $0,1,2,3$ and Latin indices the values $1,2,3$. The semicolon denotes the covariant differentiation, the indices of Cartesian tensors will be written as subscripts, the summation convention over repeated indices will be assumed and the partial differentiation will be denoted by $\partial / \partial x^{i}$.

\section{PRELIMINARIES}

In this work we shall analyse a relativistic perfect fluid characterized by the fields of particle four-flow $N^{\mu}$ and the energy-momentum tensor $T^{\mu \nu}$. These fields are usually decomposed in terms of the four-velocity $U^{\mu}$ (such that $\left.U^{\mu} U_{\mu}=c^{2}\right)$ as

$$
N^{\mu}=n U^{\mu}, \quad T^{\mu \nu}=(\epsilon+p) \frac{U^{\mu} U^{\nu}}{c^{2}}-p g^{\mu \nu}
$$

Here $n, p$ and $\epsilon$ denote the particle number density, the pressure and the energy density of the relativistic fluid, respectively. The energy density has two parts $\epsilon=\rho c^{2}\left(1+\varepsilon / c^{2}\right)$ one associated with the mass density $\rho=m n-$ where $m$ denotes the rest mass of a fluid particle - and another to the internal energy density $\rho \varepsilon$ of the gas. In the work of Chandrasekhar [2] the internal energy density $\rho \varepsilon$ is represented by $\rho \Pi$ while in the book of Weinberg [3] the energy density $\epsilon$ is simply represented by $\rho$.

The post-Newtonian approximation is a method for the solution of Einstein's field equations by considering successive approximations which are expanded in powers of $1 / c^{2}$. The starting point is the general expression for the line element $d s$ written in terms of the metric tensor $g_{\mu \nu}$, namely $d s^{2}=c^{2} d \tau^{2}=g_{\mu \nu} d x^{\mu} d x^{\nu}=g_{00}\left(d x^{0}\right)^{2}+2 g_{0 i} d x^{0} d x^{i}+$ $g_{i j} d x^{i} d x^{j}$, where $\tau$ is the proper time and $d x^{0}=c d t$. From the knowledge of the metric tensor components in a Minkowski space-time $g_{00}=1, g_{i j}=-\delta_{i j}$ and $g_{0 i}=0$ the components of the metric tensor are split as

$$
g_{00}=1+\stackrel{2}{g}_{00}+\stackrel{4}{g}_{00}+\stackrel{6}{g}_{00}+\mathcal{O}\left(c^{-8}\right), \quad g_{i j}=-\delta_{i j}+\stackrel{2}{g}_{i j}+\stackrel{4}{g}_{i j}+\mathcal{O}\left(c^{-6}\right), \quad g_{0 i}=\stackrel{3}{g}_{0 i}+\stackrel{5}{g}_{0 i}+\mathcal{O}\left(c^{-7}\right)
$$

where $\stackrel{n}{g}_{\mu \nu}$ denote the metric tensor components of order $\mathcal{O}\left(c^{-n}\right)$. Furthermore, the components of the Christoffel symbol, the Ricci tensor and the energy-momentum tensor are also split in orders $\mathcal{O}\left(c^{-n}\right)$.

The solution of Einstein's field equations leads the following expressions for the metric tensor component:1 (see Chandrasekhar and Nutku [4])

$$
\begin{aligned}
& g_{00}=1-\frac{2 U}{c^{2}}+\frac{2}{c^{4}}\left(U^{2}-2 \Phi\right)+\frac{\Psi_{00}}{c^{6}}+\mathcal{O}\left(c^{-8}\right) \\
& g_{0 i}=\frac{\Pi_{i}}{c^{3}}+\frac{\Psi_{0 i}}{c^{5}}+\mathcal{O}\left(c^{-7}\right) \\
& g_{i j}=-\left(1+\frac{2 U}{c^{2}}\right) \delta_{i j}+\frac{\Psi_{i j}}{c^{4}}+\mathcal{O}\left(c^{-6}\right)
\end{aligned}
$$

while the corresponding components of the Christoffel symbols are given in the Appendix A.

The Newtonian $U$ and the post-Newtonian $\Phi, \Pi_{i}, \Psi_{i j}, \Psi_{0 i}$ and $\Psi_{00}$ gravitational potentials that follow from

\footnotetext{
1 The correspondence of the potentials given here with those in [4] are: $\Pi_{i} \rightarrow P_{i}, \Psi_{i j} \rightarrow Q_{i j}, \Psi_{0 i} \rightarrow Q_{0 i}$ and $\Psi_{00} \rightarrow Q_{00}$
} 
Einstein's field equations are given in terms of the Poisson equations (see [4])

$$
\begin{gathered}
\nabla^{2} U=-4 \pi G \rho, \quad \nabla^{2} \Phi=-4 \pi G \rho\left(V^{2}+U+\frac{\varepsilon}{2}+\frac{3 p}{2 \rho}\right), \quad \nabla^{2} \Pi_{i}=-16 \pi G \rho V_{i}+\frac{\partial^{2} U}{\partial t \partial x^{i}}, \\
\nabla^{2} \Psi_{i j}=16 \pi G \rho\left(V_{i} V_{j}-V^{2} \delta_{i j}-2 \frac{p}{\rho} \delta_{i j}\right)-2\left(\delta_{i j} \nabla^{2}+\frac{\partial^{2}}{\partial x^{i} \partial x^{j}}\right)\left(U^{2}+2 \Phi\right)+4 \frac{\partial U}{\partial x^{i}} \frac{\partial U}{\partial x^{j}}-2 \frac{\partial^{2} U}{\partial t^{2}} \delta_{i j} \\
-\frac{\partial}{\partial t}\left(\frac{\partial \Pi_{i}}{\partial x^{j}}+\frac{\partial \Pi_{j}}{\partial x^{i}}\right), \\
\nabla^{2} \Psi_{0 i}=-16 \pi G \rho\left[V_{i}\left(V^{2}+\varepsilon+\frac{p}{\rho}+4 U\right)-\frac{\Pi_{i}}{2}\right]-10 \frac{\partial U}{\partial t} \frac{\partial U}{\partial x^{i}}-2 \frac{\partial U}{\partial x^{j}} \frac{\partial \Pi_{j}}{\partial x^{i}}+2 \Pi_{j} \frac{\partial^{2} U}{\partial x^{i} \partial x^{j}}, \\
\nabla^{2} \Psi_{00}=16 \pi G \rho\left[V^{2}\left(V^{2}+\varepsilon+\frac{p}{\rho}+4 U\right)-U^{2}-2 \Phi\right]+2 \frac{\partial U}{\partial x^{i}} \frac{\partial \Pi_{i}}{\partial t}-6\left(\frac{\partial U}{\partial t}\right)^{2}+12 \frac{\partial \Phi^{i}}{\partial x^{i}} \frac{\partial x^{i}}{\partial \Pi^{2}} \\
-12 U\left(\frac{\partial U}{\partial x^{i}}\right)^{2}+\frac{\partial \Pi_{j}}{\partial x^{i}}\left(\frac{\partial \Pi_{i}}{\partial x^{j}}-\frac{\partial \Pi_{j}}{\partial x^{i}}\right)+2 \Psi_{i j} \frac{\partial^{2} U}{\partial x^{i} \partial x^{j}} .
\end{gathered}
$$

In the above equations $V_{i}$ is the fluid hydrodynamic velocity and in the Poisson equations for $\Psi_{i j}, \Psi_{0 i}$ and $\Psi_{00}$ the gauge proposed in the work [4] was taken into account.

The components of the hydrodynamic four-velocity

$$
\left(U^{\mu}\right)=\left(\frac{d x^{0}}{d \tau}, \frac{d x^{i}}{d \tau}\right)=\left(c \frac{d t}{d \tau}, V_{i} \frac{d t}{d \tau}\right)
$$

are determined from the the expression

$$
\left(\frac{d \tau}{d t}\right)^{2}=g_{00}+\frac{2}{c} g_{0 i} V^{i}+\frac{1}{c^{2}} g_{i j} V^{i} V^{j}=1-\frac{1}{c^{2}}\left(2 U+V^{2}\right)+\frac{2}{c^{4}}\left(U^{2}-U V^{2}-2 \Phi+\Pi_{i} V^{i}\right)+\mathcal{O}\left(c^{-5}\right),
$$

which results from the line element $d s$ together with the components of the metric tensor (3) - (5). Indeed, by using the approximation $1 / \sqrt{1+x} \approx 1-x / 2+3 x^{2} / 8$ the components of the hydrodynamic four-velocity read

$$
U^{0}=c\left[1+\frac{1}{c^{2}}\left(\frac{V^{2}}{2}+U\right)+\frac{1}{c^{4}}\left(\frac{3 V^{4}}{8}+\frac{5 U V^{2}}{2}+\frac{U^{2}}{2}+2 \Phi-\Pi_{i} V_{i}\right)\right], \quad U^{i}=\frac{V_{i} U^{0}}{c} .
$$

Once the components of the four-velocity (12) are known up to order $1 / c^{4}$ we can obtain the components of the particle four-flow and energy-momentum tensor (11) in the corresponding order. They read

$$
\begin{aligned}
N^{0} & =n c\left[1+\frac{1}{c^{2}}\left(\frac{V^{2}}{2}+U\right)+\frac{1}{c^{4}}\left(\frac{3 V^{4}}{8}+\frac{5 U V^{2}}{2}+\frac{U^{2}}{2}+2 \Phi-\Pi_{i} V_{i}\right)\right], \quad N^{i}=\frac{N^{0} V_{i}}{c} . \\
T^{00} & =\rho c^{2}\left[1+\left(V^{2}+\varepsilon+2 U\right)+\frac{\rho}{c^{4}} V^{2}\left(V^{2}+\varepsilon+\frac{p}{\rho}+6 U\right)+2 U \varepsilon-2 \Pi_{i} V_{i}+2 U^{2}+4 \Phi\right], \\
T^{i 0} & =\rho c V_{i}\left\{1+\frac{1}{c^{2}}\left(V^{2}+2 U+\varepsilon+\frac{p}{\rho}\right)+\frac{1}{c^{4}}\left[V^{4}+6 V^{2} U+2 U^{2}+\left(\varepsilon+\frac{p}{\rho}\right)\left(V^{2}+2 U\right) 4 \Phi-2 \Pi_{j} V_{j}\right]\right\}-\frac{p \Pi_{i}}{c^{3}}, \\
T^{i j} & =\rho\left(V_{i} V_{j}+\frac{p}{\rho} \delta_{i j}\right)+\frac{\rho}{c^{2}}\left[\left(V^{2}+2 U+\varepsilon+\frac{p}{\rho}\right) V_{i} V_{j}-2 \frac{p}{\rho} U \delta_{i j}\right]+\frac{\rho}{c^{4}}\left\{\left[V^{4}+6 U V^{2}+2 U^{2}\right.\right. \\
& \left.\left.+\left(\varepsilon+\frac{p}{\rho}\right)\left(V^{2}+2 U\right)-2 \Pi_{i} V_{i}+4 \Phi\right] V_{i} V_{j}+\frac{p}{\rho}\left(4 U^{2} \delta_{i j}+\Psi_{i j}\right)\right\} .
\end{aligned}
$$

\section{POST-NEWTONIAN BOLTZMANN EQUATION}

In the relativistic kinetic theory a particle of a gas with rest mass $m$ is characterized by the space-time coordinates $\left(x^{\mu}\right)=(c t, \mathbf{x})$ and momentum four-vector $\left(p^{\mu}\right)=\left(p^{0}, \mathbf{p}\right)$. From the constraint that the length of the momentum four-vector is constant, i.e. $g_{\mu \nu} p^{\mu} p^{\nu}=m^{2} c^{2}$, the time component is given in terms of its spatial components by

$$
p^{0}=\frac{\sqrt{g_{00} m^{2} c^{2}+\left(g_{0 i} g_{0 j}-g_{00} g_{i j}\right) p^{i} p^{j}}-g_{0 i} p^{i}}{g_{00}} .
$$


The state of the gas in the phase space represented by the spatial coordinates $\mathbf{x}$ and momentum $\mathbf{p}$ is characterized by the one-particle distribution function $f(\mathbf{x}, \mathbf{p}, t)$ such that $d N=f(\mathbf{x}, \mathbf{p}, t) d^{3} X d^{3} P$, gives the number of particle world lines that crosses the hypersurface element represented by the three-dimensional space $d^{3} X$ on the surface $x^{0}=$ constant and with the spatial momentum four-vector contained in the cell $d^{3} P$ of the mass-shell. The one-particle distribution function is a scalar invariant and the invariant volume elements $d^{3} X$ and $d^{3} P$ are (see e.g. [9])

$$
d^{3} X=p^{0} \sqrt{-g} d^{3} x=p^{\prime 0} \sqrt{-g^{\prime}} d^{3} x^{\prime}, \quad d^{3} P=\sqrt{-g} \frac{d^{3} p}{p_{0}}=\sqrt{-g^{\prime}} \frac{d^{3} p^{\prime}}{p_{0}^{\prime}},
$$

where $g$ is the determinant of the metric tensor.

The Boltzmann equation is a non-linear integro-differential equation for the space-time evolution of the one-particle distribution function $f(\mathbf{x}, \mathbf{p}, t)$ in the phase space spanned by the spatial coordinates $\mathbf{x}$ and momentum $\mathbf{p}$ of the particles. Its expression for collisionless systems in the first post-Newtonian approximation was derived in the paper [7, 8]. Here we follow a different method for the derivation of the post-Newtonian Boltzmann equation, which was used for the determination of the Boltzmann equation in gravitational fields (see e.g. [9]).

We start by writing the one-particle distribution function $f(\mathbf{x}, \mathbf{p}, t)$ as $f\left(x^{\mu}(\tau), v_{i}(\tau)\right)$ where $\mathbf{v}=\mathbf{p} / m$ represents the particle velocity and $\tau$ the proper time along the world line of the particle. The variation of the one-particle distribution function with respect to the proper time is

$$
\frac{d f\left(x^{\mu}(\tau), v_{i}(\tau)\right)}{d \tau}=\frac{\partial f}{\partial x^{\mu}} \frac{d x^{\mu}}{d \tau}+\frac{\partial f}{\partial v_{i}} \frac{d v_{i}}{d \tau}=u^{\mu} \frac{\partial f}{\partial x^{\mu}}+\frac{\partial f}{\partial v_{i}} \frac{d v_{i}}{d t} \frac{d t}{d \tau},
$$

where $\left(u^{\mu}\right)=\left(u^{0}, u^{0} v_{i} / c\right)$ is the four-velocity of the gas particles. The corresponding expressions for $u^{0}$ and $u^{i}$ are obtained from the substitution of the hydrodynamic velocities $U^{\mu}$ and $\mathbf{V}$ by the particle velocities $u^{\mu}$ and $\mathbf{v}$ in (12), namely

$$
u^{0}=c\left[1+\frac{1}{c^{2}}\left(\frac{v^{2}}{2}+U\right)+\frac{1}{c^{4}}\left(\frac{3 v^{4}}{8}+\frac{5 U v^{2}}{2}+\frac{U^{2}}{2}+2 \Phi-\Pi_{i} v_{i}\right)\right], \quad u^{i}=\frac{v_{i} u^{0}}{c} .
$$

The first term of the second equality in (19) computed to the $1 / c^{4}$ order is

$$
u^{\mu} \frac{\partial f}{\partial x^{\mu}}=u^{0} \frac{\partial f}{\partial x^{0}}+u^{i} \frac{\partial f}{\partial x^{i}}=\left[1+\frac{1}{c^{2}}\left(\frac{v^{2}}{2}+U\right)+\frac{1}{c^{4}}\left(\frac{3 v^{4}}{8}+\frac{5 U v^{2}}{2}+\frac{U^{2}}{2}+2 \Phi-\Pi_{i} v_{i}\right)\right]\left(\frac{\partial f}{\partial t}+v_{i} \frac{\partial f}{\partial x^{i}}\right) .
$$

For the second term of the last equality in (19) we calculate first the equation of motion of the gas particles

$$
\frac{d^{2} x^{\mu}}{d \tau^{2}}+\Gamma_{\nu \lambda}^{\mu} \frac{d x^{\nu}}{d \tau} \frac{d x^{\lambda}}{d \tau}=0
$$

and compute the acceleration which follows from this equation (see Weinberg [3] )

$$
\frac{d^{2} x^{i}}{d\left(x^{0}\right)^{2}}=\left(\frac{d x^{0}}{d \tau}\right)^{-1} \frac{d}{d \tau}\left[\left(\frac{d x^{0}}{d \tau}\right)^{-1} \frac{d x^{i}}{d \tau}\right]=\left(\frac{d x^{0}}{d \tau}\right)^{-2}\left[\frac{d^{2} x^{i}}{d \tau^{2}}-\left(\frac{d x^{0}}{d \tau}\right)^{-1} \frac{d^{2} x^{0}}{d \tau^{2}} \frac{d x^{i}}{d \tau}\right]
$$

This equation can be rewritten by using (22) as

$$
\begin{aligned}
& \frac{d^{2} x^{i}}{d\left(x^{0}\right)^{2}}=\left(\frac{d x^{0}}{d \tau}\right)^{-2} \frac{d x^{\mu}}{d \tau} \frac{d x^{\nu}}{d \tau}\left[\Gamma_{\mu \nu}^{0}\left(\frac{d x^{0}}{d \tau}\right)^{-1} \frac{d x^{i}}{d \tau}-\Gamma_{\mu \nu}^{i}\right]=-\Gamma_{00}^{i}+\Gamma_{j k}^{i} \frac{d x^{j}}{d x^{0}} \frac{d x^{k}}{d x^{0}}-2 \Gamma^{i}{ }_{0 j} \frac{d x^{j}}{d x^{0}} \\
& +\frac{d x^{i}}{d x^{0}}\left[\Gamma_{00}^{0}+2 \Gamma_{0 j}^{0} \frac{d x^{j}}{d x^{0}}+\Gamma_{j k}^{0} \frac{d x^{j}}{d x^{0}} \frac{d x^{k}}{d x^{0}}\right] .
\end{aligned}
$$

If we use in (24) the expressions for the Christoffel symbol components in the Appendix A its post-Newtonian approximation up to order $1 / c^{6}$ becomes

$$
\begin{aligned}
& \frac{d^{2} x^{i}}{d t^{2}}=c^{2}\left\{-\stackrel{2}{\Gamma}^{i}{ }_{00}-\stackrel{4}{\Gamma}^{i}{ }_{00}-\stackrel{6}{\Gamma}^{i}{ }_{00}-2 \frac{v_{j}}{c}\left(\stackrel{3}{\Gamma}^{i}{ }_{0 j}+\stackrel{5}{\Gamma}_{0 j}^{i}\right)+\frac{v_{i}}{c}\left[\stackrel{3}{\Gamma}^{0}{ }_{00}+\stackrel{5}{\Gamma}_{00}^{0}+2 \frac{v_{j}}{c}\left(\stackrel{2}{\Gamma}^{0}{ }_{0 j}+\stackrel{4}{\Gamma}^{0}{ }_{0 j}\right)+\frac{v_{j} v_{k}}{c^{2}} \Gamma^{0}{ }_{j k}\right]\right. \\
& \left.-\frac{v_{j} v_{k}}{c^{2}}\left(\stackrel{2}{\Gamma}_{j k}^{i}+\stackrel{\Gamma}{\Gamma}_{j k}^{i}\right)\right\}=\frac{\partial U}{\partial x^{i}}-\frac{v_{i}}{c^{2}}\left[\frac{\partial U}{\partial t}+2 v_{j} \frac{\partial U}{\partial x^{j}}\right]-\frac{1}{c^{2}}\left(1-\frac{2 U}{c^{2}}\right)\left[2 \frac{\partial\left(U^{2}-\Phi\right)}{\partial x^{i}}+\left(2 v_{i} v_{j} \frac{\partial U}{\partial x^{j}}-v^{2} \frac{\partial U}{\partial x^{i}}\right)\right. \\
& \left.-\frac{\partial \Pi_{i}}{\partial t}-v_{j}\left(\frac{\partial \Pi_{i}}{\partial x^{j}}-\frac{\partial \Pi_{j}}{\partial x^{i}}-2 \delta_{i j} \frac{\partial U}{\partial t}\right)\right]+\frac{v_{i}}{c^{4}}\left[v_{j} v_{k} \frac{\partial \Pi_{j}}{\partial x^{k}}+v^{2} \frac{\partial U}{\partial t}\right]+\frac{2 v_{j}}{c^{4}}\left[\Pi_{i} \frac{\partial U}{\partial x^{j}}+\frac{1}{2} \frac{\partial \Psi_{i 0}}{\partial x^{j}}-\frac{1}{2} \frac{\partial \Psi_{j 0}}{\partial x^{i}}+\frac{1}{2} \frac{\partial \Psi_{i j}}{\partial t}\right] \\
& +\frac{v_{i}}{c^{4}}\left[\Pi_{j} \frac{\partial U}{\partial x^{j}}-2 \frac{\partial \Phi}{\partial t}-4 v_{j} \frac{\partial \Phi}{\partial x^{j}}\right]+\frac{v_{j} v_{k}}{2 c^{4}}\left[2 \frac{\partial \Psi_{i j}}{\partial x^{k}}-\frac{\partial \Psi_{j k}}{\partial x^{i}}\right]+\frac{1}{c^{4}}\left[\Pi_{i} \frac{\partial U}{\partial t}+\frac{\partial \Psi_{i 0}}{\partial t}-\frac{1}{2} \frac{\partial \Psi_{00}}{\partial x^{i}}+\Psi_{i j} \frac{\partial U}{\partial x^{j}}\right] .
\end{aligned}
$$


The Boltzmann equation in the second post-Newtonian approximation follows from (19) by taking into account (21) and (25) and reads

$$
\begin{aligned}
& {\left[\frac{\partial f}{\partial t}+v_{i} \frac{\partial f}{\partial x^{i}}+\frac{\partial f}{\partial v_{i}} \frac{\partial U}{\partial x^{i}}\right]\left[1+\frac{1}{c^{2}}\left(\frac{v^{2}}{2}+U\right)+\frac{1}{c^{4}}\left(\frac{3 v^{4}}{8}+\frac{5 v^{2} U}{2}+\frac{U^{2}}{2}+2 \Phi-\Pi_{j} v_{j}\right)\right]} \\
& +\frac{1}{c^{2}} \frac{\partial f}{\partial v_{i}}\left\{\left[1+\frac{1}{c^{2}}\left(\frac{v^{2}}{2}-U\right)\right]\left[v_{j}\left(\frac{\partial \Pi_{i}}{\partial x^{j}}-\frac{\partial \Pi_{j}}{\partial x^{i}}\right)-2 v_{i} \frac{\partial U}{\partial t}+\frac{\partial \Pi_{i}}{\partial t}-2 \frac{\partial\left(U^{2}-\Phi\right)}{\partial x^{i}}-2 v_{i} v_{j} \frac{\partial U}{\partial x^{j}}+v^{2} \frac{\partial U}{\partial x^{i}}\right]\right. \\
& -v_{i}\left[1+\frac{1}{c^{2}}\left(\frac{v^{2}}{2}+U\right)\right]\left[\frac{\partial U}{\partial t}+2 v_{j} \frac{\partial U}{\partial x^{j}}\right]+\frac{v_{j} v_{k}}{2 c^{2}}\left[2 \frac{\partial \Psi_{i j}}{\partial x^{k}}-\frac{\partial \Psi_{j k}}{\partial x^{i}}\right]+\frac{1}{c^{2}}\left[\Pi_{i} \frac{\partial U}{\partial t}+\frac{\partial \Psi_{i 0}}{\partial t}-\frac{1}{2} \frac{\partial \Psi_{00}}{\partial x^{i}}+\Psi_{i j} \frac{\partial U}{\partial x^{j}}\right] \\
& \left.+\frac{v_{i}}{c^{2}}\left[v_{j} v_{k} \frac{\partial \Pi_{j}}{\partial x^{k}}+v^{2} \frac{\partial U}{\partial t}\right]+\frac{2 v_{j}}{c^{2}}\left[\Pi_{i} \frac{\partial U}{\partial x^{j}}+\frac{1}{2} \frac{\partial \Psi_{i 0}}{\partial x^{j}}-\frac{1}{2} \frac{\partial \Psi_{j 0}}{\partial x^{i}}+\frac{1}{2} \frac{\partial \Psi_{i j}}{\partial t}\right]+\frac{v_{i}}{c^{2}}\left[\Pi_{j} \frac{\partial U}{\partial x^{j}}-2 \frac{\partial \Phi}{\partial t}-4 v_{j} \frac{\partial \Phi}{\partial x^{j}}\right]\right\}=\mathcal{Q}(f, f) .
\end{aligned}
$$

Here we have introduced the so-called collision operator of the Boltzmann equation $\mathcal{Q}(f, f)$, which refers to the binary collision of the particles and is given in terms of an integral of the product of two particle distribution functions at collision. In the first post-Newtonian approximation the Boltzmann equation reduces to

$$
\begin{gathered}
{\left[\frac{\partial f}{\partial t}+v_{i} \frac{\partial f}{\partial x^{i}}+\frac{\partial f}{\partial v_{i}} \frac{\partial U}{\partial x^{i}}\right]\left[1+\frac{1}{c^{2}}\left(\frac{v^{2}}{2}+U\right)\right]+\frac{1}{c^{2}} \frac{\partial f}{\partial v_{i}}\left\{v_{j}\left(\frac{\partial \Pi_{i}}{\partial x^{j}}-\frac{\partial \Pi_{j}}{\partial x^{i}}\right)-2 v_{i} \frac{\partial U}{\partial t}\right.} \\
\left.+\frac{\partial \Pi_{i}}{\partial t}-2 \frac{\partial\left(U^{2}-\Phi\right)}{\partial x^{i}}-2 v_{i} v_{j} \frac{\partial U}{\partial x^{j}}+v^{2} \frac{\partial U}{\partial x^{i}}-v_{i}\left[\frac{\partial U}{\partial t}+2 v_{j} \frac{\partial U}{\partial x^{j}}\right]\right\}=\mathcal{Q}(f, f),
\end{gathered}
$$

which is the expression obtained in [8] if we identify there $\phi \rightarrow-U, \xi_{i} \rightarrow-\Pi_{i}$ and $\psi \rightarrow-2 \Phi$.

\section{POST-NEWTONIAN MAXWELL-JÜTTNER DISTRIBUTION FUNCTION}

At equilibrium the collision term of the Boltzmann vanishes, since it refers to the difference of the number of particles that enter and leave the volume element in the phase space. An important consequence is that one can determine the equilibrium distribution function from the condition that the collision term vanishes at equilibrium (for more details one is referred to e.g. see [9]). In the relativistic kinetic theory the equilibrium distribution function is the so-called Maxwell-Jüttner distribution function which reads

$$
f(\mathbf{x}, \mathbf{p}, t)=\frac{n}{4 \pi m^{2} c k T K_{2}(\zeta)} \exp \left(-\frac{p^{\mu} U_{\mu}}{k T}\right) .
$$

Here $k$ is the Boltzmann constant, $T$ the absolute temperature and $K_{2}(\zeta)$ the modified Bessel function of the second kind which depends on the relativistic parameter $\zeta$. The relativistic parameter $\zeta=m c^{2} / k T$ represents the ratio of the rest energy of the gas particles $m c^{2}$ and the thermal energy of the gas $k T$ and in the non-relativistic limiting case $\zeta \gg 1$ while in the ultra-relativistic limiting case $\zeta \ll 1$.

For the determination of the Maxwell-Jüttner distribution function in the second post-Newtonian approximation we begin with the determination of its exponential term. We introduce the so-called peculiar velocity $\mathcal{V}_{i}=v_{i}-V_{i}$ - which is the difference of the particle velocity and the gas velocity, i.e. it refers to the particle velocity in the gas frame - and get the following relationship by using the components of the metric tensor $g_{\mu \nu}$, fluid $U^{\mu}$ and particle $u^{\mu}=p^{\mu} / m$ four-velocities

$$
\begin{aligned}
& \frac{g_{\mu \nu} p^{\mu} U^{\nu}}{k T}=\frac{m}{k T}\left\{c^{2}+\frac{\mathcal{V}^{2}}{2}+\frac{1}{c^{2}}\left[2 U \mathcal{V}^{2}+\frac{V^{2} \mathcal{V}^{2}}{2}+\frac{\left(V_{i} \mathcal{V}_{i}\right)^{2}}{2}+\left(V_{i} \mathcal{V}_{i}\right) \mathcal{V}^{2}+\frac{3 \mathcal{V}^{4}}{8}\right]+\frac{1}{c^{4}}\left[3 U^{2} \mathcal{V}^{2}+4 U V^{2} \mathcal{V}^{2}\right.\right. \\
& +4 U\left(V_{i} \mathcal{V}_{i}\right)^{2}+8 U\left(V_{i} \mathcal{V}_{i}\right) \mathcal{V}^{2}+3 U \mathcal{V}^{4}+\frac{V^{4} \mathcal{V}^{2}}{2}+V^{2}\left(V_{i} \mathcal{V}_{i}\right)^{2}+2 V^{2}\left(V_{i} \mathcal{V}_{i}\right) \mathcal{V}^{2}+\frac{3 V^{2} \mathcal{V}^{4}}{4}+\left(V_{i} \mathcal{V}_{i}\right)^{3} \\
& \left.\left.+\frac{9\left(V_{i} \mathcal{V}_{i}\right)^{2} \mathcal{V}^{2}}{4}+\frac{3\left(V_{i} \mathcal{V}_{i}\right) \mathcal{V}^{4}}{2}+\frac{5 \mathcal{V}^{6}}{16}-\Pi_{i} \mathcal{V}_{i}\left(V_{i} \mathcal{V}_{i}\right)-\Pi_{i} V_{i} \mathcal{V}^{2}-\Pi_{i} \mathcal{V}_{i} \mathcal{V}^{2}+2 \Phi \mathcal{V}^{2}-\frac{\mathcal{V}_{i} \mathcal{V}_{j} \Psi_{i j}}{2}\right]\right\}
\end{aligned}
$$

Next up to the $1 / c^{4}$ order the modified Bessel function of second kind reads [12]

$$
\frac{1}{K_{2}(\zeta)}=\sqrt{\frac{2 m c^{2}}{\pi k T}} e^{\frac{m c^{2}}{k T}}\left(1-\frac{15 k T}{8 m c^{2}}+\frac{345(k T)^{2}}{128 m^{2} c^{4}}\right) .
$$


The Maxwell-Jüttner distribution function in the second post-Newtonian approximation is obtained from (28) (30) by considering the approximation $e^{-x} \approx 1-x+x^{2} / 2$ for the $1 / c^{2}-$ terms in the exponential, yielding

$$
\begin{aligned}
& f=\frac{n}{(2 \pi m k T)^{\frac{3}{2}}} e^{-\frac{m \mathcal{V}^{2}}{2 k T}}\left\{1-\frac{1}{c^{2}}\left[\frac{15 k T}{8 m}+\frac{m\left(V_{i} \mathcal{V}_{i}\right)^{2}}{2 k T}+\frac{2 m \mathcal{V}^{2}}{k T}+\frac{3 m \mathcal{V}^{4}}{8 k T}+\frac{m V^{2} \mathcal{V}^{2}}{2 k T}+\frac{m\left(V_{i} \mathcal{V}_{i}\right) \mathcal{V}^{2}}{k T}\right]\right. \\
& +\frac{1}{c^{4}}\left[\frac{2 m^{2} U^{2} \mathcal{V}^{4}}{(k T)^{2}}+\frac{m^{2} U V^{2} \mathcal{V}^{4}}{(k T)^{2}}+\frac{3 m^{2} U \mathcal{V}^{6}}{4(k T)^{2}}+\frac{m^{2} U\left(V_{i} \mathcal{V}_{i}\right)^{2} \mathcal{V}^{2}}{(k T)^{2}}+\frac{2 m^{2} U\left(V_{i} \mathcal{V}_{i}\right) \mathcal{V}^{4}}{(k T)^{2}}+\frac{3 m^{2} V^{2} \mathcal{V}^{6}}{16(k T)^{2}}+\frac{m^{2} V^{4} \mathcal{V}^{4}}{8(k T)^{2}}\right. \\
& +\frac{m^{2}\left(V_{i} \mathcal{V}_{i}\right)^{4}}{8(k T)^{2}}+\frac{m^{2} V^{2}\left(V_{i} \mathcal{V}_{i}\right)^{2} \mathcal{V}^{2}}{4(k T)^{2}}+\frac{m^{2} V^{2}\left(V_{i} \mathcal{V}_{i}\right) \mathcal{V}^{4}}{2(k T)^{2}}+\frac{m^{2}\left(V_{i} \mathcal{V}_{i}\right)^{3} \mathcal{V}^{2}}{2(k T)^{2}}+\frac{11 m^{2}\left(V_{i} \mathcal{V}_{i}\right)^{2} \mathcal{V}^{4}}{16(k T)^{2}}+\frac{3 m^{2}\left(V_{i} \mathcal{V}_{i}\right) \mathcal{V}^{6}}{8(k T)^{2}} \\
& +\frac{9 m^{2} \mathcal{V}^{8}}{128(k T)^{2}}+\frac{345(k T)^{2}}{128 m^{2}}-\frac{3 m U^{2} \mathcal{V}^{2}}{k T}-\frac{4 m U V^{2} \mathcal{V}^{2}}{k T}-\frac{4 m U\left(V_{i} \mathcal{V}_{i}\right)^{2}}{k T}-\frac{8 m U\left(V_{i} \mathcal{V}_{i}\right) \mathcal{V}^{2}}{k T}-\frac{3 m U \mathcal{V}^{4}}{k T}+\frac{m \Pi \mathcal{V}_{i}\left(V_{i} \mathcal{V}_{i}\right)}{k T} \\
& -\frac{m V^{4} \mathcal{V}^{2}}{2 k T}-\frac{m V^{2}\left(V_{i} \mathcal{V}_{i}\right)^{2}}{k T}-\frac{2 m V^{2}\left(V_{i} \mathcal{V}_{i}\right) \mathcal{V}^{2}}{k T}-\frac{3 m V^{2} \mathcal{V}^{4}}{4 k T}-\frac{m\left(V_{i} \mathcal{V}_{i}\right)^{3}}{k T}-\frac{9 m\left(V_{i} \mathcal{V}_{i}\right)^{2} \mathcal{V}^{2}}{4 k T}-\frac{3 m\left(V_{i} \mathcal{V}_{i}\right) \mathcal{V}^{4}}{2 k T}-\frac{5 m \mathcal{V}^{6}}{16 k T} \\
& \left.+\frac{m \Pi_{i} V_{i} \mathcal{V}^{2}}{k T}+\frac{m \Pi_{i} \mathcal{V}_{i} \mathcal{V}^{2}}{k T}-\frac{2 m \Phi \mathcal{V}^{2}}{k T}+\frac{m \mathcal{V}_{i} \mathcal{V}_{j} \Psi_{i j}}{2 k T}+\frac{15 U \mathcal{V}^{2}}{4}+\frac{15 V^{2} \mathcal{V}^{2}}{16}+\frac{15\left(V_{i} \mathcal{V}_{i}\right)^{2}}{16}+\frac{15\left(V_{i} \mathcal{V}_{i}\right) \mathcal{V}^{2}}{8}+\frac{45 \mathcal{V}^{4}}{64}\right]
\end{aligned}
$$

In the first post-Newtonian approximation the Maxwell-Jüttner distribution function becomes [10]

$$
f=\frac{n}{(2 \pi m k T)^{\frac{3}{2}}} e^{-\frac{m \mathcal{V}^{2}}{2 k T}}\left\{1-\frac{1}{c^{2}}\left[\frac{15 k T}{8 m}+\frac{m\left(V_{i} \mathcal{V}_{i}\right)^{2}}{2 k T}+\frac{2 m U \mathcal{V}^{2}}{k T}+\frac{3 m \mathcal{V}^{4}}{8 k T}+\frac{m V^{2} \mathcal{V}^{2}}{2 k T}+\frac{m\left(V_{i} \mathcal{V}_{i}\right) \mathcal{V}^{2}}{k T}\right]\right\}
$$

In the kinetic theory of relativistic gases the particle four-flow and the energy-momentum tensor are given in terms of the one-particle distribution function $f(\mathbf{x}, \mathbf{v}, t)$ (see e.g. [9]) by

$$
N^{\mu}=m^{4} c \int u^{\mu} f \frac{\sqrt{-g} d^{3} u}{u_{0}}, \quad T^{\mu \nu}=m^{4} c \int u^{\mu} u^{\nu} f \frac{\sqrt{-g} d^{3} u}{u_{0}} .
$$

The transformation of the differential elements $d^{3} u=d u^{1} d u^{2} d u^{3}=|J| d v_{1} d v_{2} d v_{3}=|J| d^{3} v$ is given by the Jacobian matrix which follows from (20) by computing of the derivatives $J=\partial\left(u^{1}, u^{2}, u^{3}\right) / \partial\left(v_{1}, v_{2}, v_{3}\right)$. The final expression for the transformation of the differential elements in the second post-Newtonian approximation reads

$$
d^{3} u=\left[1+\frac{1}{c^{2}}\left(\frac{5 v^{2}}{2}+3 U\right)+\frac{1}{c^{4}}\left(\frac{35 v^{4}}{8}+\frac{9 U^{2}}{2}+6 \Phi+\frac{35 U v^{2}}{2}-4 \Pi_{i} v_{i}\right)\right] d^{3} v
$$

Moreover, from the expressions for the components of the metric tensor we have that

$$
u_{0}=\left(g_{00} u^{0}+g_{0 i} u^{i}\right)=u^{0}\left[1-2 \frac{U}{c^{2}}+\frac{1}{c^{4}}\left(2 U^{2}-4 \Phi+\Pi_{i} v_{i}\right)\right], \quad \sqrt{-g}=1+\frac{2 U}{c^{2}}-\frac{1}{c^{4}}\left(U^{2}+2 \Phi+\frac{\Psi_{k k}}{2}\right)
$$

Hence the integration element up to the $1 / c^{4}$ order which follows from (34) and (35) reads

$$
\begin{gathered}
\frac{\sqrt{-g} d^{3} u}{u_{0}}=\left[1+\frac{1}{c^{2}}\left(\frac{5 v^{2}}{2}+7 U\right)+\frac{1}{c^{4}}\left(\frac{35 v^{4}}{8}+\frac{55 U v^{2}}{2}+\frac{43 U^{2}}{2}+8 \Phi-\frac{\Psi_{k k}}{2}-5 \Pi_{i} v_{i}\right)\right] \frac{d^{3} v}{u^{0}} \\
=\left\{1+\frac{1}{c^{2}}\left[2 v^{2}+6 U\right]+\frac{1}{c^{4}}\left[3 v^{4}+20 U v^{2}+15 U^{2}+6 \Phi-4 \Pi_{i} v_{i}-\frac{\Psi_{k k}}{2}\right]\right\} \frac{d^{3} v}{c} .
\end{gathered}
$$

From the knowledge of the Maxwell-Jüttner distribution function (31) and of the integration element (36) it is possible to determine the components of the particle four-flow and energy-momentum tensor in the second postNewtonian approximation. For the integration it is necessary to introduce the peculiar velocity $\mathcal{V}_{i}=v_{i}-V_{i}$ in (36), spherical coordinates and express the integral element as $d^{3} \mathcal{V}=\mathcal{V}^{2} \sin \theta d \theta d \varphi$, where $0 \leq \theta \leq \pi$ and $0 \leq \varphi \leq 2 \pi$.

The insertion of the Maxwell-Jüttner distribution function (31) and the integration element (36) into the definition of the particle four-flow (33) 1 with subsequent integration of the resulting equation leads to (13). Note that for the integration one makes use of the table of integrals given in the Appendix B. The components of the energy-momentum 
tensor $(33)_{2}$ follow in the same manner and read

$$
\begin{aligned}
T^{00}= & \rho c^{2}\left[1+\frac{1}{c^{2}}\left(V^{2}+2 U+\frac{3 k T}{2 m}\right)+\frac{1}{c^{4}}\left(V^{4}+6 U V^{2}+2 U^{2}+\frac{5 k T V^{2}}{2 m}+\frac{15(k T)^{2}}{8 m^{2}}+\frac{3 k T U}{m}-2 \Pi_{i} V_{i}+4 \Phi\right)\right], \\
T^{0 i}= & \rho c V_{i}\left[1+\frac{1}{c^{2}}\left(V^{2}+2 U+\frac{5 k T}{2 m}\right)+\frac{1}{c^{4}}\left(V^{4}+6 U V^{2}+2 U^{2}+\frac{5 k T V^{2}}{2 m}+\frac{15(k T)^{2}}{8 m^{2}}+\frac{5 k T U}{m}\right.\right. \\
& \left.\left.-2 \Pi_{j} V_{j}+4 \Phi\right)\right]-\frac{k T \Pi_{i}}{m c^{4}} \\
T^{i j}= & \rho\left(V_{i} V_{j}+\frac{k T}{m} \delta_{i j}\right)+\frac{\rho}{c^{2}}\left[\left(V^{2}+2 U+\frac{3 k T_{0}}{2 m}\right) V_{i} V_{j}-\frac{2 k T U}{m} \delta_{i j}\right]+\frac{\rho}{c^{4}}\left[\left(V^{4}+6 U V^{2}+2 U^{2}\right.\right. \\
& \left.\left.+\frac{5 k T V^{2}}{2 m}+\frac{15(k T)^{2}}{8 m^{2}}+\frac{5 k T U}{m}-2 \Pi_{k} V_{k}+4 \Phi\right) V_{i} V_{j}+\frac{4 k T U^{2}}{m} \delta_{i j}+\frac{k T \Psi_{i j}}{m}\right]
\end{aligned}
$$

If we make use of the thermal equation of state and the expression of the specific internal energy, namely

$$
p=\frac{\rho k T}{m}, \quad \varepsilon=\frac{3 k T}{2 m}\left(1+\frac{5 k T}{4 m c^{2}}\right)
$$

the above expressions for the components of the energy-momentum tensor match the ones given by (14) - (16).

\section{POST-NEWTONIAN EULERIAN HYDRODYNAMIC EQUATIONS}

The Eulerian hydrodynamic equations in the first post-Newtonian approximation were determined by Chandrasekhar [2] and Weinberg [3] from a macroscopic description based on the equations of conservation of the particle four-flow $N^{\mu}$ and energy-momentum tensor $T^{\mu \nu}$, namely

$$
N_{; \mu}^{\mu}=\frac{\partial N^{\mu}}{\partial x^{\mu}}+\Gamma_{\mu \lambda}^{\mu} N^{\lambda}=0, \quad T_{; \nu}^{\mu \nu}=\frac{\partial T^{\mu \nu}}{\partial x^{\nu}}+\Gamma_{\nu \lambda}^{\mu} T^{\lambda \nu}+\Gamma_{\nu \lambda}^{\nu} T^{\mu \lambda}=0,
$$

while the second post-Newtonian Eulerian hydrodynamic equations were obtained by Chandrasekhar and Nutku [4]. Here we shall obtain the second post-Newtonian Eulerian hydrodynamic equations from the Boltzmann equation (26).

\section{A. Mass density hydrodynamic equation}

We begin with the determination of the mass density hydrodynamic equation and for that end we multiply the Boltzmann equation (26) by $m^{4} \sqrt{-g} d^{3} u / u_{0}$, use the Maxwell-Jüttner distribution function (31), the integration element (36) and integrate the resulting equation, yielding

$$
\begin{aligned}
& \frac{\partial}{\partial t}\left\{\rho\left[1+\frac{1}{c^{2}}\left(\frac{V^{2}}{2}+U\right)+\frac{1}{c^{4}}\left(\frac{3 V^{4}}{8}+\frac{5 V^{2} U}{2}+\frac{U^{2}}{2}+2 \Phi-\Pi_{j} V_{j}\right)\right]\right\} \\
& +\frac{\partial}{\partial x^{i}}\left\{\rho V_{i}\left[1+\frac{1}{c^{2}}\left(\frac{V^{2}}{2}+U\right)+\frac{1}{c^{4}}\left(\frac{3 V^{4}}{8}+\frac{5 V^{2} U}{2}+\frac{U^{2}}{2}+2 \Phi-\Pi_{j} V_{j}\right)\right]\right\} \\
& +2 \frac{\rho}{c^{2}}\left(\frac{\partial U}{\partial t}+V_{i} \frac{\partial U}{\partial x^{i}}\right)+\frac{\rho}{c^{4}}\left(\frac{\partial U}{\partial t}+V_{i} \frac{\partial U}{\partial x^{i}}\right)\left(V^{2}-4 U\right)-\frac{\rho}{c^{4}}\left[2\left(\frac{\partial \Phi}{\partial t}+V_{i} \frac{\partial \Phi}{\partial x^{i}}\right)+\frac{1}{2}\left(\frac{\partial \Psi_{k k}}{\partial t}+V_{i} \frac{\partial \Psi_{k k}}{\partial x^{i}}\right)\right]=0 .
\end{aligned}
$$

The Newtonian continuity equation follows from the above equation by neglecting all terms in $1 / c^{2}$ and $1 / c^{4}$

$$
\frac{\partial \rho}{\partial t}+\frac{\partial \rho V_{i}}{\partial x^{i}}=0
$$

The first post-Newtonian approximation for the continuity follows from (42) neglecting all $1 / c^{4}$ terms

$$
\frac{\partial}{\partial t}\left\{\rho\left[1+\frac{1}{c^{2}}\left(\frac{V^{2}}{2}+U\right)\right]\right\}+\frac{\partial}{\partial x^{i}}\left\{\rho V_{i}\left[1+\frac{1}{c^{2}}\left(\frac{V^{2}}{2}+U\right)\right]\right\}+2 \frac{\rho}{c^{2}}\left(\frac{\partial U}{\partial t}+V_{i} \frac{\partial U}{\partial x^{i}}\right)=0 .
$$


The last term above can be rewritten as

$$
2 \frac{\rho}{c^{2}}\left(\frac{\partial U}{\partial t}+V_{i} \frac{\partial U}{\partial x^{i}}\right)=\frac{2}{c^{2}}\left(\frac{\partial \rho U}{\partial t}+\frac{\partial \rho U V_{i}}{\partial x^{i}}\right)-\frac{2 U}{c^{2}} \underline{\left(\frac{\partial \rho}{\partial t}+\frac{\partial \rho V_{i}}{\partial x^{i}}\right)}
$$

where for the underlined term vanishes thanks to the Newtonian continuity equation (44). Hence it follows the final form of the continuity equation in the first post-Newtonian approximation

$$
\frac{\partial \rho_{*}}{\partial t}+\frac{\partial \rho_{*} V_{i}}{\partial x^{i}}=0, \quad \text { where } \quad \rho_{*}=\rho\left[1+\frac{1}{c^{2}}\left(\frac{V^{2}}{2}+3 U\right)\right] .
$$

The notation for the mass density $\rho_{*}$ was introduced by Fock [13] and the above equation corresponds to eq. (117) of Chandrasekhar [2].

In order to get the continuity equation in the second post-Newtonian approximation we have to transform the underlined terms in (42) as follows. The first underlined term can be rewritten as

$$
2 \frac{\rho}{c^{2}}\left(\frac{\partial U}{\partial t}+V_{i} \frac{\partial U}{\partial x^{i}}\right)=\frac{2}{c^{2}}\left(\frac{\partial \rho U}{\partial t}+\frac{\partial \rho V_{i} U}{\partial x^{i}}\right)+\frac{U}{c^{4}}\left[\left(\frac{\partial \rho V^{2}}{\partial t}+\frac{\partial \rho V^{2} V_{i}}{\partial x^{i}}\right)+6\left(\frac{\partial \rho U}{\partial t}+\frac{\partial \rho U V_{i}}{\partial x^{i}}\right)\right],
$$

where the expression for the continuity equation in the first post-Newtonian approximation (44) was used. The second underlined term can be transformed according to

$$
\begin{gathered}
\frac{\rho}{c^{4}}\left(\frac{\partial U}{\partial t}+V_{i} \frac{\partial U}{\partial x^{i}}\right)\left(V^{2}-4 U\right)=\frac{1}{c^{4}}\left\{\frac{\partial \rho U V^{2}}{\partial t}+\frac{\partial \rho U V^{2} V_{i}}{\partial x^{i}}-U\left(\frac{\partial \rho V^{2}}{\partial t}+\frac{\partial \rho V^{2} V_{i}}{\partial x^{i}}\right)\right. \\
\left.-4 U\left[\frac{\partial \rho U}{\partial t}+\frac{\partial \rho U V_{i}}{\partial x^{i}}-U\left(\frac{\partial \rho}{\partial t}+\frac{\partial \rho V_{i}}{\partial x^{i}}\right)\right]\right\} .
\end{gathered}
$$

Here we note that for the above underlined term the Newtonian continuity equation (44) can be used so that this term vanishes. Now by adding the two equation (47) and (48) we get

$$
\begin{aligned}
& 2 \frac{\rho}{c^{2}}\left(\frac{\partial U}{\partial t}+V_{i} \frac{\partial U}{\partial x^{i}}\right)+\frac{\rho}{c^{4}}\left(\frac{\partial U}{\partial t}+V_{i} \frac{\partial U}{\partial x^{i}}\right)\left(V^{2}-4 U\right)=\frac{2}{c^{2}}\left(\frac{\partial \rho U}{\partial t}+\frac{\partial \rho U V_{i}}{\partial x^{i}}\right)+\frac{1}{c^{4}}\left\{\frac{\partial \rho U V^{2}}{\partial t}+\frac{\partial \rho U V^{2} V_{i}}{\partial x^{i}}\right. \\
& \left.+2 U\left(\frac{\partial \rho U}{\partial t}+\frac{\partial \rho U V_{i}}{\partial x^{i}}\right)\right\}=\frac{2}{c^{2}}\left(\frac{\partial \rho U}{\partial t}+\frac{\partial \rho U V_{i}}{\partial x^{i}}\right)+\frac{1}{c^{4}}\left\{\frac{\partial \rho U V^{2}}{\partial t}+\frac{\partial \rho U V^{2} V_{i}}{\partial x^{i}}+\left(\frac{\partial \rho U^{2}}{\partial t}+\frac{\partial \rho U^{2} V_{i}}{\partial x^{i}}\right)\right\}+\mathcal{O}\left(c^{-6}\right) .
\end{aligned}
$$

The last underlined term in (42) can be written as

$$
\begin{aligned}
-\frac{\rho}{c^{4}}\left[2\left(\frac{\partial \Phi}{\partial t}+V_{i} \frac{\partial \Phi}{\partial x^{i}}\right)+\frac{1}{2}\left(\frac{\partial \Psi_{k k}}{\partial t}+V_{i} \frac{\partial \Psi_{k k}}{\partial x^{i}}\right)\right]=- & \frac{1}{c^{4}}\left[\frac{\partial \rho\left(2 \Phi+\frac{\Psi_{k k}}{2}\right)}{\partial t}+\frac{\partial \rho V_{i}\left(2 \Phi+\frac{\Psi_{k k}}{2}\right)}{\partial x^{i}}\right] \\
& +\frac{1}{c^{4}}\left(2 \Phi+\frac{\Psi_{k k}}{2}\right) \underline{\left(\frac{\partial \rho}{\partial t}+\frac{\partial \rho V_{i}}{\partial x^{i}}\right)}
\end{aligned}
$$

where the underlined term above vanishes thanks to the Newtonian continuity equation (44).

The continuity equation in the second post-Newtonian approximation is obtained from (42) by using (47)- (50), yielding

$$
\frac{\partial \widetilde{\rho}}{\partial t}+\frac{\partial \widetilde{\rho} V_{i}}{\partial x^{i}}=0, \quad \text { where } \quad \tilde{\rho}=\rho\left[1+\frac{1}{c^{2}}\left(\frac{V^{2}}{2}+3 U\right)+\frac{1}{c^{4}}\left(\frac{3}{8} V^{4}+\frac{7}{2} U V^{2}+\frac{3}{2} U^{2}-\frac{1}{2} \Psi_{k k}-\Pi_{i} V_{i}\right)\right] .
$$

The expression for $\widetilde{\rho}$ for the mass density in the second post-Newtonian approximation corresponds to eq. (53) of Chandrasekhar and Nutku [4] and was determined from the consideration that the volume integral of $\rho U^{0} \sqrt{-g}$ is constant which is a consequence of the particle four-flow conservation equation.

\section{B. Mass-energy density hydrodynamic equation}

The mass-energy density hydrodynamic equation is obtained by applying the same methodology, i.e. the Boltzmann equation (26) is multiplied by $m^{4} u^{0} \sqrt{-g} d^{3} u / u_{0}$, the Maxwell-Jüttner distribution function (31) and the integration 
element (36) are used and the resulting equation integrated. The result is

$$
\begin{array}{r}
\frac{\partial}{\partial t}\left\{\rho\left[1+\frac{1}{c^{2}}\left(V^{2}+2 U+\varepsilon\right)+\frac{1}{c^{4}}\left(V^{4}+6 V^{2} U+2 U^{2}+V^{2}\left(\varepsilon+\frac{p}{\rho}\right)+2 U \varepsilon+4 \Phi-2 \Pi_{j} V_{j}\right)\right]\right\} \\
+\frac{\partial}{\partial x^{i}}\left\{\rho V _ { i } \left[1+\frac{1}{c^{2}}\left(V^{2}+2 U+\varepsilon+\frac{p}{\rho}\right)+\frac{1}{c^{4}}\left(V^{4}+6 V^{2} U+2 U^{2}+4 \Phi+\left(2 U+V^{2}\right)\left(\varepsilon+\frac{p}{\rho}\right)\right.\right.\right. \\
\left.\left.\left.-2 \Pi_{j} V_{j}\right)\right]-\frac{p \Pi_{i}}{c^{4}}\right\}+\frac{\rho}{c^{2}} \frac{\partial U}{\partial t}+\frac{\rho}{c^{4}}\left[\left(\frac{3 k T}{2 m}+2 U+2 V^{2}\right) \frac{\partial U}{\partial t}-3\left(\frac{\partial U^{2}}{\partial t}+V_{i} \frac{\partial U^{2}}{\partial x^{i}}\right)+\Pi_{i} \frac{\partial U}{\partial x^{i}}\right. \\
\left.-\frac{1}{2}\left(\frac{\partial \Psi_{k k}}{\partial t}+V_{i} \frac{\partial \Psi_{k k}}{\partial x^{i}}\right)-\left(4 \frac{\partial \Phi}{\partial t}+6 V_{i} \frac{\partial \Phi}{\partial x^{i}}\right)+V_{i} V_{j} \frac{\partial \Pi_{i}}{\partial x^{j}}\right]=0 .
\end{array}
$$

By neglecting all terms in $1 / c^{2}$ and $1 / c^{4}$ we get the Newtonian continuity equation (43).

The first post-Newtonian approximation to the mass-energy hydrodynamic equation is obtained from (52) by neglecting the $1 / c^{4}$ terms, yielding

$$
\frac{\partial \sigma}{\partial t}+\frac{\partial \sigma V_{i}}{\partial x^{i}}+\frac{1}{c^{2}}\left(\rho \frac{\partial U}{\partial t}-\frac{\partial p}{\partial t}\right)=0, \quad \text { where } \quad \sigma=\rho\left[1+\frac{1}{c^{2}}\left(V^{2}+2 U+\varepsilon+\frac{p}{\rho}\right)\right] .
$$

The abbreviation $\sigma$ was introduced by Chandrasekhar [2] and this equation corresponds to the eq. (64) of that work.

If we introduce the the abbreviations

$$
\begin{aligned}
& \varphi=V^{2}+U+\frac{\varepsilon}{2}+\frac{3 p}{2 \rho} \\
& \widetilde{\sigma}=\rho\left\{1+\frac{1}{c^{2}}\left(V^{2}+2 U+\varepsilon+\frac{p}{\rho}\right)+\frac{1}{c^{4}}\left[V^{4}+6 V^{2} U-U^{2}+2 U \varepsilon+V^{2}\left(\varepsilon+\frac{p}{\rho}\right)-\Pi_{i} V_{i}-\frac{1}{2} \Psi_{k k}\right]\right\} .
\end{aligned}
$$

we can rewrite the second approximation to the mass-energy hydrodynamic equation (52) in the following form

$$
\frac{\partial \widetilde{\sigma}}{\partial t}+\frac{\partial \widetilde{\sigma} V_{i}}{\partial x^{i}}+\frac{1}{c^{2}}\left(\rho \frac{\partial U}{\partial t}-\frac{\partial p}{\partial t}\right)+\frac{2 \rho}{c^{4}}\left[\varphi \frac{\partial U}{\partial t}-V_{i} \frac{\partial \Phi}{\partial x^{i}}+\frac{1}{\rho} \frac{\partial p U V_{i}}{\partial x^{i}}-\frac{V_{i}}{2} \frac{\partial \Pi_{i}}{\partial t}\right]=0 .
$$

In this equation the term

$$
\frac{1}{c^{4}}\left(3 U^{2}+4 \Phi+\frac{\Psi_{k k}}{2}\right)\left[\frac{\partial \rho}{\partial t}+\frac{\partial \rho V_{i}}{\partial x^{i}}\right]-\frac{\Pi_{i}}{c^{4}}\left[\frac{\partial \rho V_{i}}{\partial t}+\frac{\partial \rho V_{i} V_{j}}{\partial x^{j}}+\frac{\partial p}{\partial x^{i}}-\rho \frac{\partial U}{\partial x^{i}}\right] .
$$

was neglected, since the Newtonian continuity equation (43) and the momentum hydrodynamic equation (59) for the first and the second terms within the brackets above can be used, respectively.

Equation (56) has not been derived in the work of Chandrasekhar and Nutku [4] but we shall see that it is important to determine the post-Newtonian hydrodynamic equation for the internal energy density.

\section{Momentum density hydrodynamic equation}

The hydrodynamic equation for the momentum density is obtained from the multiplication of Boltzmann equation (26) by $m^{4} u^{i} \sqrt{-g} d^{3} u / u_{0}$ and integration of the resulting equation, taking into account the Maxwell-Jüttner distribution function (31) and the integration element (36). Up to the first post-Newtonian approximation we get

$$
\begin{aligned}
& \frac{\partial}{\partial t}\left\{\rho\left[1+\frac{1}{c^{2}}\left(V^{2}+2 U+\varepsilon+\frac{p}{\rho}\right)\right] V_{i}\right\}+\frac{\partial}{\partial x^{j}}\left\{\rho V_{i} V_{j}\left[1+\frac{1}{c^{2}}\left(V^{2}+2 U+\varepsilon+\frac{p}{\rho}\right)\right]\right\}+\frac{\partial}{\partial x^{i}}\left[p\left(1-\frac{2 U}{c^{2}}\right)\right] \\
- & \rho\left[1+\frac{1}{c^{2}}\left(2 V^{2}-2 U+\varepsilon-\frac{p}{\rho}\right)\right] \frac{\partial U}{\partial x^{i}}+4 \frac{\rho}{c^{2}} V_{i}\left(\frac{\partial U}{\partial t}+V_{j} \frac{\partial U}{\partial x^{j}}\right)-\frac{\rho}{c^{2}}\left[\frac{\partial \Pi_{i}}{\partial t}+V_{j}\left(\frac{\partial \Pi_{i}}{\partial x^{j}}-\frac{\partial \Pi_{j}}{\partial x^{i}}\right)+2 \frac{\partial \Phi}{\partial x^{i}}\right]=0 .
\end{aligned}
$$

By neglecting the $1 / c^{2}$ terms we get the Newtonian momentum density hydrodynamic equation

$$
\frac{\partial \rho V_{i}}{\partial t}+\frac{\partial \rho V_{i} V_{j}}{\partial x^{j}}+\frac{\partial p}{\partial x^{i}}-\rho \frac{\partial U}{\partial x^{i}}=0
$$


The following terms can be rewritten in the equivalent forms

$$
\begin{aligned}
& \frac{\partial}{\partial x^{i}}\left[p\left(1-\frac{2 U}{c^{2}}\right)\right]=\frac{\partial p}{\partial x^{i}}\left(1-\frac{2 U}{c^{2}}\right)-\frac{2 p}{c^{2}} \frac{\partial U}{\partial x^{i}} \\
& 4 \frac{\rho}{c^{2}} V_{i}\left(\frac{\partial U}{\partial t}+V_{j} \frac{\partial U}{\partial x^{j}}\right)=\frac{4}{c^{2}}\left(\frac{\partial \rho U V_{i}}{\partial t}+\frac{\partial \rho U V_{i} V_{j}}{\partial x^{j}}\right)-\frac{4}{c^{2}} U\left(\frac{\partial \rho V_{i}}{\partial t}+\frac{\partial \rho V_{i} V_{j}}{\partial x^{j}}\right) \\
& \quad=\frac{4}{c^{2}}\left(\frac{\partial \rho U V_{i}}{\partial t}+\frac{\partial \rho U V_{i} V_{j}}{\partial x^{j}}\right)-\frac{4}{c^{2}} U\left(\frac{\partial p}{\partial x^{i}}-\frac{\partial U}{\partial x^{i}}\right)+\mathcal{O}\left(c^{-4}\right) \\
& \frac{\rho}{c^{2}}\left(\frac{\partial \Pi_{i}}{\partial t}+V_{j} \frac{\partial \Pi_{i}}{\partial x^{j}}\right)=\frac{1}{c^{2}}\left(\frac{\partial \rho \Pi_{i}}{\partial t}+\frac{\partial \rho \Pi_{i} V_{j}}{\partial x^{j}}\right)-\frac{\Pi_{i}}{c^{2}}\left(\frac{\partial \rho}{\partial t}+\frac{\partial \rho V_{j}}{\partial x^{j}}\right)=\frac{1}{c^{2}}\left(\frac{\partial \rho \Pi_{i}}{\partial t}+\frac{\partial \rho \Pi_{i} V_{j}}{\partial x^{j}}\right)+\mathcal{O}\left(c^{-4}\right)
\end{aligned}
$$

where the Newtonian hydrodynamic equations for the momentum density (59) and for the mass density (43) were used in the equations (61) and (62), respectively. By collecting the above results the momentum density hydrodynamic equation (58) in the first post-Newtonian approximation can be rewritten as

$$
\frac{\partial \rho \mathfrak{V}_{i}}{\partial t}+\frac{\partial \rho \mathfrak{V}_{i} V_{j}}{\partial x^{j}}+\frac{\partial p}{\partial x^{i}}\left[1+\frac{2 U}{c^{2}}\right]-\rho \frac{\partial U}{\partial x^{i}}\left[1+\frac{2}{c^{2}}\left(V^{2}+U+\frac{\varepsilon}{2}+\frac{3 p}{2 \rho}\right)\right]+\frac{\rho}{c^{2}}\left(V_{j} \frac{\partial \Pi_{j}}{\partial x^{i}}-2 \frac{\partial \Phi}{\partial x^{i}}\right)=0 .
$$

Here we have introduced the following abbreviation for the momentum density

$$
\rho \mathfrak{V}_{i}=\rho V_{i}\left[1+\frac{1}{c^{2}}\left(V^{2}+6 U+\varepsilon+\frac{p}{\rho}\right)\right]-\frac{\rho}{c^{2}} \Pi_{i} .
$$

A more familiar equation for the momentum density hydrodynamic equation (63) is obtained when the material time derivative $d / d t=\partial / \partial t+V_{i} \partial / \partial x^{i}$ is introduced. After some rearrangements this equation becomes

$$
\begin{array}{r}
\rho \frac{d V_{i}}{d t}+\frac{\partial p}{\partial x^{i}}\left[1-\frac{1}{c^{2}}\left(V^{2}+4 U+\varepsilon+\frac{p}{\rho}\right)\right]-\rho \frac{\partial U}{\partial x^{i}}\left[1+\frac{1}{c^{2}}\left(V^{2}-4 U\right)\right] \\
-\frac{\rho}{c^{2}}\left[2 \frac{\partial \Phi}{\partial x^{i}}+\frac{d \Pi_{i}}{d t}-V_{j} \frac{\partial \Pi_{j}}{\partial x^{i}}+V_{i}\left(\frac{\partial U}{\partial t}-\frac{1}{\rho} \frac{\partial p}{\partial t}-4 \frac{d U}{d t}\right)\right]=0 .
\end{array}
$$

As was pointed in [4] the hydrodynamic equation for the momentum density in the second post-Newtonian approximation follows after a long calculation, yielding

$$
\begin{aligned}
\frac{\partial \rho \widetilde{\mathfrak{V}}_{i}}{\partial t} & +\frac{\partial \rho \widetilde{\mathfrak{V}}_{i} V_{j}}{\partial x^{j}}+\frac{\partial p}{\partial x^{i}}\left[1+\frac{2 U}{c^{2}}-\frac{1}{c^{4}}\left(U^{2}+2 \Phi+\frac{\Psi k k}{2}\right)\right]-\rho \frac{\partial U}{\partial x^{i}}\left\{1+\frac{2}{c^{2}}\left(V^{2}+U+\frac{\varepsilon}{2}+\frac{3 p}{2 \rho}\right)+\frac{2}{c^{4}}\left[V^{4}+5 U V^{2}\right.\right. \\
& \left.\left.\left.-\frac{3 U^{2}}{2}+\Phi+\left(V^{2}+U\right)\left(\varepsilon+\frac{p}{\rho}\right)-\Pi_{i} V_{i}-\frac{\Psi_{k k}}{4}\right]\right\}+\frac{\rho}{c^{2}}\left(V_{j} \frac{\partial \Pi_{j}}{\partial x^{i}}-2 \frac{\partial \Phi}{\partial x^{i}}\right)\left[1+\frac{1}{c^{2}}\left(V^{2}+4 U+\varepsilon+\frac{p}{\rho}\right)\right]\right] \\
& +\frac{\rho}{2 c^{4}}\left(\frac{\partial \Psi_{00}}{\partial x^{i}}+2 V_{j} \frac{\partial \Psi_{0 j}}{\partial x^{i}}+V_{j} V_{k} \frac{\partial \Psi_{j k}}{\partial x^{i}}\right)=0
\end{aligned}
$$

where the abbreviation for the momentum density was introduced

$$
\begin{aligned}
& \rho \widetilde{\mathfrak{V}}_{i}=\rho V_{i}\left\{1+\frac{1}{c^{2}}\left(V^{2}+6 U+\varepsilon+\frac{p}{\rho}\right)+\frac{1}{c^{4}}\left[V^{4}+10 V^{2} U+13 U^{2}+2 \Phi-2 \Pi_{i} V_{i}-\frac{\Psi_{k k}}{2}\right.\right. \\
& \left.\left.+\left(V^{2}+6 U\right)\left(\varepsilon+\frac{p}{\rho}\right)\right]\right\}-\frac{\rho}{c^{2}} \Pi_{i}\left[1+\frac{1}{c^{2}}\left(V^{2}+4 U+\varepsilon+\frac{p}{\rho}\right)\right]-\frac{\rho}{c^{4}}\left(\Psi_{0 i}+\Psi_{i j} V_{j}\right) .
\end{aligned}
$$

\section{Total energy density hydrodynamic equation}

The total energy density of the gas is the sum of its internal $\rho \varepsilon$ and kinetic $\rho V^{2} / 2$ energy densities. Its hydrodynamic equation can be obtained by subtracting the continuity equation (43) from the mass-energy hydrodynamic equation (56), yielding

$$
\begin{aligned}
& \frac{1}{c^{2}}\left\{\frac{\partial}{\partial t}\left[\rho\left(\frac{V^{2}}{2}+\varepsilon\right)+\frac{\rho}{c^{2}}\left[\frac{5}{8} V^{4}+\frac{5}{2} V^{2} U-\frac{5}{2} U^{2}+2 \varepsilon U+V^{2}\left(\varepsilon+\frac{p}{\rho}\right)\right]\right]\right. \\
& +\frac{\partial}{\partial x^{i}}\left[\rho V_{i}\left(\frac{V^{2}}{2}+\varepsilon\right)+\frac{\rho V_{i}}{c^{2}}\left[\frac{5}{8} V^{4}+\frac{5}{2} V^{2} U-\frac{5}{2} U^{2}+2 \varepsilon U+V^{2}\left(\varepsilon+\frac{p}{\rho}\right)\right]\right] \\
& \left.+\frac{\partial p V_{i}}{\partial x^{i}}-\rho V_{i} \frac{\partial U}{\partial x^{i}}-U\left(\frac{\partial \rho}{\partial t}+\frac{\partial \rho V_{i}}{\partial x^{i}}\right)+\frac{2 \rho}{c^{2}}\left[\varphi \frac{\partial U}{\partial t}-V_{i} \frac{\partial \Phi}{\partial x^{i}}+\frac{1}{\rho} \frac{\partial p U V_{i}}{\partial x^{i}}-\frac{V_{i}}{2} \frac{\partial \Pi_{i}}{\partial t}\right]\right\}=0 .
\end{aligned}
$$


Note that the total energy density hydrodynamic equation is of order $\mathcal{O}\left(c^{-2}\right)$, meaning that the post-Newtonian corrections to the resulting equation corresponds to the first post-Newtonian approximation.

The hydrodynamic equation for the internal energy density in the first post-Newtonian approximation is obtained from (68) by eliminating the time derivative of the mass density $\rho$ and hydrodynamic velocity $V_{i}$ taking into account the first post-Newtonian hydrodynamic equations for the mass density (46) and momentum density (63). After some rearrangements we get that

$$
\rho \frac{d \varepsilon}{d t}+p\left(1-\frac{5}{3} \frac{V^{2}}{c^{2}}\right) \frac{\partial V_{i}}{\partial x^{i}}+\frac{\rho V_{i}}{c^{2}}\left(V^{2}+\frac{2}{3} \varepsilon\right)\left[\frac{\partial U}{\partial x^{i}}-\frac{1}{\rho} \frac{\partial p}{\partial x^{i}}\right]-\frac{V^{2}}{c^{2}} \frac{\partial p}{\partial t}+\frac{\rho(2 \varepsilon-5 U)}{c^{2}} \frac{d U}{d t}=0 .
$$

Without the terms in $c^{-2}$ this equation reduces to the Newtonian internal energy density hydrodynamic equation for an Eulerian fluid, namely

$$
\rho \frac{d \varepsilon}{d t}+p \frac{\partial V_{i}}{\partial x^{i}}=0
$$

\section{CONCLUSIONS}

In this work a kinetic theory of relativistic gases in the presence of gravitational fields within the second postNewtonian approximation was developed. The Boltzmann equation, the equilibrium Maxwell-Jüttner distribution function and the hydrodynamic equations were found in the same approximation. The expressions for the components of the particle four-flow, energy-momentum tensor and the hydrodynamic equations for the mass, mass-energy and momentum densities correspond to those obtained from a phenomenology theory.

\section{APPENDIX A}

In this appendix we give the components of the Christoffel symbols corresponding to the components of the metric tensor (3) - (5).

$$
\begin{aligned}
& \stackrel{3}{\Gamma}_{00}^{0}=-\frac{1}{c^{3}} \frac{\partial U}{\partial t}, \quad \stackrel{5}{\Gamma}_{00}^{0}=\frac{1}{c^{5}}\left(\Pi_{i} \frac{\partial U}{\partial x^{i}}-2 \frac{\partial \Phi}{\partial t}\right), \quad \stackrel{2}{\Gamma}_{0 i}^{0}=-\frac{1}{c^{2}} \frac{\partial U}{\partial x^{i}}, \quad \stackrel{4}{\Gamma}_{0 i}^{0}=-\frac{2}{c^{4}} \frac{\partial \Phi}{\partial x^{i}} \\
& \stackrel{2}{\Gamma}_{00}^{i}=-\frac{1}{c^{2}} \frac{\partial U}{\partial x^{i}}, \quad \stackrel{4}{\Gamma}_{00}^{i}=\frac{2}{c^{4}} \frac{\partial\left(U^{2}-\Phi\right)}{\partial x^{i}}-\frac{1}{c^{4}} \frac{\partial \Pi_{i}}{\partial t}, \quad \stackrel{3}{\Gamma}_{i j}^{0}=\frac{1}{2 c^{3}}\left(\frac{\partial \Pi_{i}}{\partial x^{j}}+\frac{\partial \Pi_{j}}{\partial x^{i}}+2 \frac{\partial U}{\partial t} \delta_{i j}\right) \\
& \stackrel{2}{\Gamma}_{j k}^{i}=\frac{1}{c^{2}}\left(\frac{\partial U}{\partial x^{j}} \delta_{i k}+\frac{\partial U}{\partial x^{k}} \delta_{i j}-\frac{\partial U}{\partial x^{i}} \delta_{j k}\right), \quad \stackrel{3}{\Gamma}_{0 i}^{j}=\frac{1}{2 c^{3}}\left(\frac{\partial \Pi_{i}}{\partial x^{j}}-\frac{\partial \Pi_{j}}{\partial x^{i}}+2 \frac{\partial U}{\partial t} \delta_{i j}\right) . \\
& \stackrel{4}{\Gamma}_{j k}^{i}=-\frac{1}{2 c^{4}}\left(\frac{\partial \Psi_{i j}}{\partial x^{k}}+\frac{\partial \Psi_{i k}}{\partial x^{j}}-\frac{\partial \Psi_{j k}}{\partial x^{i}}\right)-\frac{1}{c^{4}}\left(\frac{\partial U^{2}}{\partial x^{k}} \delta_{i j}+\frac{\partial U^{2}}{\partial x^{j}} \delta_{i k}-\frac{\partial U^{2}}{\partial x^{i}} \delta_{j k}\right) . \\
& \stackrel{5}{\Gamma}_{0 j}^{i}=-\frac{1}{c^{5}} \Pi_{i} \frac{\partial U}{\partial x^{j}}+\frac{U}{c^{5}}\left(\frac{\partial \Pi_{i}}{\partial x^{j}}-\frac{\partial \Pi_{j}}{\partial x^{i}}-2 \frac{\partial U}{\partial t} \delta_{i j}\right)-\frac{1}{2 c^{5}}\left(\frac{\partial \Psi_{0 i}}{\partial x^{j}}-\frac{\partial \Psi_{0 j}}{\partial x^{i}}+\frac{\partial \Psi_{i j}}{\partial t}\right) . \\
& \stackrel{\Gamma}{\Gamma}_{00}^{i}=\frac{1}{2 c^{6}} \frac{\partial \Psi_{00}}{\partial x^{i}}-\frac{1}{c^{6}} \frac{\partial \Psi_{0 i}}{\partial t}-\frac{\Pi_{i}}{c^{6}} \frac{\partial U}{\partial t}-\frac{4 U}{c^{6}}\left(\frac{\partial U^{2}}{\partial x^{i}}-\frac{\partial \Phi}{\partial x^{i}}\right)+\frac{2 U}{c^{6}} \frac{\partial \Pi_{i}}{\partial t}-\frac{\Psi_{i j}}{c^{6}} \frac{\partial U}{\partial x^{j}} .
\end{aligned}
$$

\section{APPENDIX B}

For the integration of the equations in Section [V] we have used the following expressions from the kinetic theory of gases (see e.g. [14])

$$
\begin{aligned}
& I_{n}=\int \mathcal{V}^{n} e^{-\frac{m \mathcal{V}^{2}}{k T}} d \mathcal{V}=\frac{1}{2} \Gamma\left(\frac{n+1}{2}\right)\left(\frac{k T}{m}\right)^{\frac{n+1}{2}} \quad \Gamma(n+1)=n \Gamma(n), \quad \Gamma(1)=1, \quad \Gamma\left(\frac{1}{2}\right)=\sqrt{\pi}, \\
& \int e^{-\frac{m \mathcal{V}^{2}}{k T_{0}}} \mathcal{V}_{i} \mathcal{V}_{j} d^{3} \mathcal{V}=\frac{I_{2}}{3} \delta_{i j}, \quad \int e^{-\frac{m \mathcal{V}^{2}}{k T_{0}}} \mathcal{V}_{i} \mathcal{V}_{j} \mathcal{V}_{k} \mathcal{V}_{l} d^{3} \mathcal{V}=\frac{I_{4}}{15}\left[\delta_{i j} \delta_{k l}+\delta_{i k} \delta_{j l}+\delta_{i l} \delta_{j k}\right], \\
& \int e^{-\frac{m \mathcal{V}^{2}}{k T_{0}}} \mathcal{V}_{i} \mathcal{V}_{j} \mathcal{V}_{k} \mathcal{V}_{l} \mathcal{V}_{n} \mathcal{V}_{n} d^{3} \mathcal{V}=\frac{I_{6}}{105}\left[\delta_{i j}\left(\delta_{k l} \delta_{m n}+\delta_{k m} \delta_{l n}+\delta_{k n} \delta_{l m}\right)+\delta_{i k}\left(\delta_{j l} \delta_{m n}+\delta_{j m} \delta_{l n}+\delta_{j n} \delta_{l m}\right)\right. \\
& \left.+\delta_{i l}\left(\delta_{j k} \delta_{m n}+\delta_{j m} \delta_{k n}+\delta_{j n} \delta_{k m}\right)+\delta_{i m}\left(\delta_{j k} \delta_{l n}+\delta_{j l} \delta_{k n}+\delta_{j n} \delta_{k l}\right)+\delta_{i n}\left(\delta_{j k} \delta_{l m}+\delta_{j l} \delta_{k m}+\delta_{j m} \delta_{k l}\right)\right] .
\end{aligned}
$$


[1] A. Einstein, L. Infeld and B. Hoffmann, The gravitational equations and the problem of motion, Ann. of Math. 39, 65 (1938).

[2] S. Chandrasekhar, The post-Newtonian equations of hydrodynamics in general relativity, Ap. J. 142, 1488 (1965).

[3] S. Weinberg, Gravitation and cosmology. Principles and applications of the theory of relativity (Wiley, New York, 1972).

[4] S. Chandrasekhar and Y. Nutku, The second post-Newtonian equations of hydrodynamics in general relativity, Ap. J. 158, 55 (1969).

[5] J. C. Maxwell, On the dynamical theory of gases, Phil. Trans. R. Soc. London 157, 49 (1867).

[6] D. Enskog, Bermerkungen zu einer Fundamentalgleichung in der kinetischen Gastheorie, Phys. Z. 12, 534 (1911).

[7] V. Rezania and Y. Sobouti, Liouville's equation in post Newtonian approximation I. Static solutions, Astron. Astrophys. 354, 1110 (2000).

[8] C. A. Agón, J. F. Pedraza and J. Ramos-Caro, Kinetic theory of collisionless self-gravitating gases: Post-Newtonian polytropes, Phys. Rev. D 83, 123007 (2011).

[9] C. Cercignani and G. M. Kremer, The relativistic Boltzmann equation: theory and applications (Birkhäuser, Basel, 2002).

[10] G. M. Kremer, M. G. Richarte and K. Weber, Self-gravitating systems of ideal gases in the 1PN approximation, Phys. Rev. D 93, 064073 (2016).

[11] S. Chandrasekhar, Conservation laws in general relativity and in the post-Newtonian approximations, Ap. J. 158, 45 (1969).

[12] M. Abramowitz and I. A. Stegun, Handbook of mathematical functions (Dover, New York, 1968).

[13] V. Fock, The theory of space time and gravitation (Pergamon Press, London, 1959).

[14] G. M. Kremer, An introduction to the Boltzmann equation and transport processes in gases (Springer, Berlin, 2010). 